\title{
Inequality in mortality between Black and White Americans by age, place, and cause and in comparison to Europe, 1990 to 2018
}

\author{
Hannes Schwandt ${ }^{a} \oplus$, Janet Currie ${ }^{b, 1} \oplus$, Marlies Bärc, James Banks ${ }^{\mathrm{d}, \mathrm{e}}$, Paola Bertolif ${ }^{\mathrm{f}}$, Aline Bütikofer ${ }^{\mathrm{g}}$, Sarah Cattan ${ }^{\mathrm{d}}$, \\ Beatrice Zong-Ying Chao ${ }^{\mathrm{a}}{ }^{\infty}$, Claudia Costa ${ }^{\mathrm{h}}{ }^{\odot}$, Libertad Gonzálezi, Veronica Grembij, Kristiina Huttunen ${ }^{\mathrm{k}}$, \\ René Karadakic $^{\mathrm{g}}{ }^{\circ}$, Lucy Kraftman ${ }^{\mathrm{d}}$, Sonya Krutikova ${ }^{\mathrm{d}}$, Stefano Lombardil ${ }^{1}{ }^{\circ}$, Peter Redler ${ }^{\mathrm{m}}$, Carlos Riumallo-Herl ${ }^{\mathrm{n}}$,

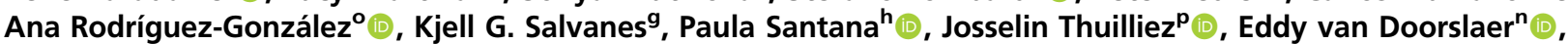 \\ Tom Van Ourti ${ }^{\mathrm{n}} \odot$, Joachim $\mathrm{K}_{\text {. Winter }}^{\mathrm{m}}{ }^{\odot}$, Bram Wouterse ${ }^{\mathrm{c}}$, and Amelie Wuppermann ${ }^{q}$
}

aSchool of Education and Social Policy, Northwestern University, Evanston, IL 60208; bepartment of Economics, Princeton University, Princeton, NJ 08540;

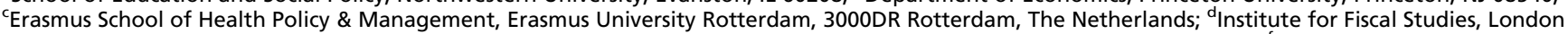
WC1E 7AE, United Kingdom; ${ }^{e}$ Department of Economics, University of Manchester, Manchester M13 9PL, United Kingdom; ${ }^{f}$ Department of Economics, University of Verona, 37129 Verona, Italy; ${ }^{9}$ Department of Economics, Norwegian School of Economics, Bergen, 5045, Norway; ${ }^{h}$ Centre of Studies in Geography and Spatial Planning, University of Coimbra, 3004-531, Coimbra, Portugal; 'Department of Economics and Business, Universitat Pompeu Fabra, 08005 Barcelona, Spain; ${ }^{j}$ Department of Economics, Management, and Quantitative Methods, University of Milan, 20122 Milano, Italy; ${ }^{\mathrm{k}}$ Department of Economics, Aalto University School of Business, 02150 Espoo, Finland; 'VATT Institute for Economic Research, 00100 Helsinki, Finland; 'mDepartment of Economics, University of Munich, 80539 Munich, Germany; ${ }^{n}$ Erasmus School of Economics, Erasmus University Rotterdam, 3000DR Rotterdam, The Netherlands; ${ }^{\circ}$ Department of Economics, Lund University, SE-220 07 Lund, Sweden; ${ }^{\mathrm{P}}$ CNRS, Centre d'économie de la Sorbonne, Université Paris 1, 75013 Paris, France; and ${ }^{9}$ Department of Economics, University of Halle, 06108 Halle (Saale), Germany

Edited by Kenneth W. Wachter, University of California, Berkeley, CA, and approved August 15, 2021 (received for review March 18, 2021)

\begin{abstract}
Although there is a large gap between Black and White American life expectancies, the gap fell $48.9 \%$ between 1990 and 2018, mainly due to mortality declines among Black Americans. We examine agespecific mortality trends and racial gaps in life expectancy in highand low-income US areas and with reference to six European countries. Inequalities in life expectancy are starker in the United States than in Europe. In 1990, White Americans and Europeans in highincome areas had similar overall life expectancy, while life expectancy for White Americans in low-income areas was lower. However, since then, even high-income White Americans have lost ground relative to Europeans. Meanwhile, the gap in life expectancy between Black Americans and Europeans decreased by 8.3\%. Black American life expectancy increased more than White American life expectancy in all US areas, but improvements in lower-income areas had the greatest impact on the racial life expectancy gap. The causes that contributed the most to Black Americans' mortality reductions included cancer, homicide, HIV, and causes originating in the fetal or infant period. Life expectancy for both Black and White Americans plateaued or slightly declined after 2012, but this stalling was most evident among Black Americans even prior to the COVID-19 pandemic. If improvements had continued at the 1990 to 2012 rate, the racial gap in life expectancy would have closed by 2036. European life expectancy also stalled after 2014. Still, the comparison with Europe suggests that mortality rates of both Black and White Americans could fall much further across all ages and in both high-income and low-income areas.
\end{abstract}

life expectancy | racial divide | area-level socioeconomic status | international comparison | age-specific mortality

$\mathbf{R}$ ecent events, notably the Black Lives Matter movement and the disproportionate impact of the COVID-19 pandemic on the Black population, have highlighted the persistent gap in life expectancy between Black Americans and other Americans $(1,2)$. In 2018, the gap in life expectancy between Black and White Americans was $3.6 \mathrm{y}$. However, there have also been tremendous improvements in life expectancy among Black Americans relative to White Americans over time and especially since 1990 (3-7). Much of the highly publicized recent research investigating changes in inequality in life expectancy and mortality in the United States over the past $30 \mathrm{y}$ highlights inequalities in adult mortality across educational and income groups (8-23).
This paper discusses the evolution of inequalities in mortality between Black and White Americans from 1990 to 2018 through the lens of place. There are two innovations: First, following several recent studies $(1,6,24-31)$, we examine the evolution of mortality rates among Black and White Americans by age and county poverty rates. This analysis allows us to see whether racial gaps have evolved differently in higher- and lower-income parts of the United States. Trends in age-specific mortality rates provide insights into whether changes in life expectancy are specific to certain age groups, for example, people over 65 who qualify for Medicare, which in turn may provide additional insight into possible mechanisms.

Second, we benchmark these developments against trends in mortality inequality across high- and low-income places in a set

\section{Significance}

From 1990 to 2018, the Black-White American life expectancy gap fell $\mathbf{4 8 . 9 \%}$ and mortality inequality decreased, although progress stalled after 2012 as life expectancy plateaued. Had improvements continued at the 1990 to 2012 rate, the racial gap in life expectancy would have closed by 2036 . Despite decreasing mortality inequality, income-based life expectancy gaps remain starker in the United States than in European countries. At the same time, European mortality improved strongly and even those U.S. populations with the longest life spans-White Americans living in the highest-income areas-experience higher mortality at all ages than Europeans in high-income areas in 2018. Hence, mortality rates of both Black and White Americans could fall much further in both high-income and low-income areas.

Author contributions: H.S., J.C., J.B., S.C., S.K., and K.G.S. conceived the overall comparative project; H.S. and J.C. designed research; H.S., J.C., and B.Z.-Y.C. performed research; M.B., P.B., A.B., S.C., C.C., L.G., V.G., K.H., R.K., L.K., S.L., P.R., C.R.-H., A.R.-G., P.S., J.T. E.v.D., T.V.O., J.K.W., B.W., and A.W. provided European mortality data in a format similar to the US data; and H.S. and J.C. wrote the paper.

The authors declare no competing interest.

This article is a PNAS Direct Submission.

This open access article is distributed under Creative Commons Attribution-NonCommercialNoDerivatives License 4.0 (CC BY-NC-ND).

${ }^{1}$ To whom correspondence may be addressed. Email: jcurrie@princeton.edu.

This article contains supporting information online at https://www.pnas.org/lookup/suppl/ doi:10.1073/pnas.2104684118/-/DCSupplemental.

Published September 28, 2021 
of six prosperous European countries. This comparison offers several potential insights, such as whether mortality in higher-income parts of the United States is more similar to that of European countries or whether both high- and low-income US places tend to lag behind. It also provides additional perspective on the gaps between Black and White Americans, allowing us to ask, for example, if only Black Americans fall short of a European benchmark or if the mechanisms driving lower life expectancy in the United States also affect White Americans.

Our analysis proceeds by first ranking counties by their poverty rate in each year and then grouping counties into clusters that each account for about $5 \%$ of the population (Materials and Methods). A key advantage of this approach is that we can examine all deaths, whereas information on income and completed education is not available for every person. We also avoid problems due to changes in the education distribution over time. For example, high school dropouts in the United States have become an increasingly small and more negatively selected group as high school completion and college attendance have become more normative (32-35). A limitation of our approach is that we cannot examine inequalities within small areas. Set against this limitation is evidence that lowincome Americans live longer in high-income areas than in lowincome ones (12), so that mortality across geographic areas is of independent interest. Our approach allows us to see whether changes in mortality occur in both high- and low-income areas or are driven largely by improvements in lower-income areas.

A second advantage of this geographical approach is that it can be easily adapted to examine mortality inequality in other countries using a similar framework. We examine trends in mortality inequality in six wealthy European countries using methods identical to the US analysis. Mortality inequality in these countries is of interest in its own right but also serves as a useful baseline for considering developments in the United States, contributing to a growing body of comparative literature on mortality differentials (6-39). Our main analysis focuses on six countries (England, France, Germany, the Netherlands, Norway, and Spain) for which consistent mortality data by geographic areas exist for the entire time period. All six are prosperous countries with well-developed health care and social welfare systems. The experience of these countries provides some insight into questions such as how low US mortality rates could fall given current medical standards; whether increasing gaps in life expectancy between the United States and Europe are driven only by lower-income areas or whether higherincome areas are also falling behind; and finally, whether mortality among Black Americans declined only relative to White Americans or whether it also declined relative to a European life expectancy benchmark.

\section{Results}

Our main results for age-specific mortality rates are shown in Figs. $1-4$, representing the age groups 0 to 4,5 to 19,20 to 64 , and 65 to 79. Each figure has three panels showing estimates for the years 1990, 2005, and 2018 and contains three heavy lines representing mortality rates for Black Americans, White Americans, and Europeans as defined in our study. Each marker on these figures represents a county group representing about $5 \%$ of a country's population. The lines drawn through the markers are simply least squares linear regression lines through the points. The data for each marker and the slopes of the regression lines are shown in SI Appendix, Tables S1-S4, along with $P$ values for whether the slopes of the regression lines are equal to 0 , whether the slopes for lines representing Black and White persons are equal, and whether the slopes of the White American and European lines are equal. The figures also show fainter gray lines corresponding to mortality rates in the individual European countries. Further details are provided in Materials and Methods and in SI Appendix.

The biggest takeaway from Fig. 1 is the huge gap in mortality between Black and White children aged 0 to 4 in 1990 and the equally stunning narrowing of the gap between Black and White child deaths over the subsequent decades. In 1990, 4.2 out of every 1,000 Black children aged 0 to 4 died compared to 1.82 per 1,000 White children. In 2018, the rates had fallen to 2.31 per 1,000 for young Black children and 1.13 per 1,000 for White children. Viewed as a percentage, the progress is less impressive-in 1990, 2.3 Black children died for every White child, while in 2018, 2.04 Black children died for every White child. However, the increase in the total number of lives saved was much greater for young Black children, resulting in much closer absolute mortality rates in 2018. Much of the improvement in Black child mortality rates happened between 1990 and 2005, with only slow progress from 2005 to 2018. Mortality rates for White children aged 0 to 4 also fell throughout the period, although at a slower rate.

Mortality improvements among young Black children occurred across the entire economic spectrum of US locations, although reductions were somewhat stronger in the highest-income areas, which led to an increase in mortality inequality for Black American children. Mortality inequality among White children decreased slightly. Hence, the strong reduction in overall inequality in mortality for young children aged 0 to 4 in high- and low-income areas that has been previously reported $(6,25-27)$ is due to the higher concentration of Black children in low-income areas combined with the large reduction in mortality rates among young Black children. Overall, despite strong improvements, mortality among young Black children remained substantially higher and more unequally distributed between high- and low-income places compared to White children.

Inequality in mortality among young children aged 0 to 4 in Europe was lower than in the United States in all years, and the European gradient between mortality rates and area poverty was almost entirely flat in 2018 (see SI Appendix, Table S1 for numerical values of the gradients). Fig. 1 shows that in 1990, the average US White mortality rate for the 0 to 4 age group was similar to the European rate, although deaths were more unequally distributed in the United States. Specifically, US death rates among White children aged 0 to 4 were lower than European rates in the highest-income areas and higher in the lowestincome areas in 1990. By 2005, mortality for White children aged 0 to 4 had pulled away from European levels and was uniformly higher than in Europe in both high- and low-income areas. This trend continued to 2018 .

Fig. 2 tells a broadly similar story for children aged 5 to 19 . The biggest difference is that even in 1990, White mortality rates for children aged 5 to 19 were higher than European rates in all but the highest-income US places. By 2005, the gap between European children and White American children had become wider than the gap between Black and White American children, which suggests that all American children in this age group suffered high levels of mortality relative to the lower potential mortality rates implied by the European baseline.

Fig. 3 shows trends in mortality for adults aged 20 to 64 . (See SI Appendix for a further split into ages 20 to 49 and 50 to 64.) Focusing first on the US story, the three panels show that Black and White prime-age adult mortality (aged 20 to 64) converged sharply over time driven primarily by a rapid fall in Black mortality, especially in the lowest-income areas. In the highest-income areas of the United States, the gap in Black-White American death rates had fallen to 0.7 deaths per 1,000 by 2018, while in lower-income places it was still 1.47 (SI Appendix, Table S3). The comparison with Europe is striking. In 1990 and in 2005, White Americans in the highest-income area had mortality rates very similar to Europeans, while Black Americans suffered much higher mortality even in high-income areas. By 2018, European mortality rates were uniformly lower: the gap between Europeans and White Americans was generally larger than the gap between White and Black Americans. In large part this pattern is due to stagnation in US White mortality rates. The lower European mortality rates 


\section{Age 0-4}
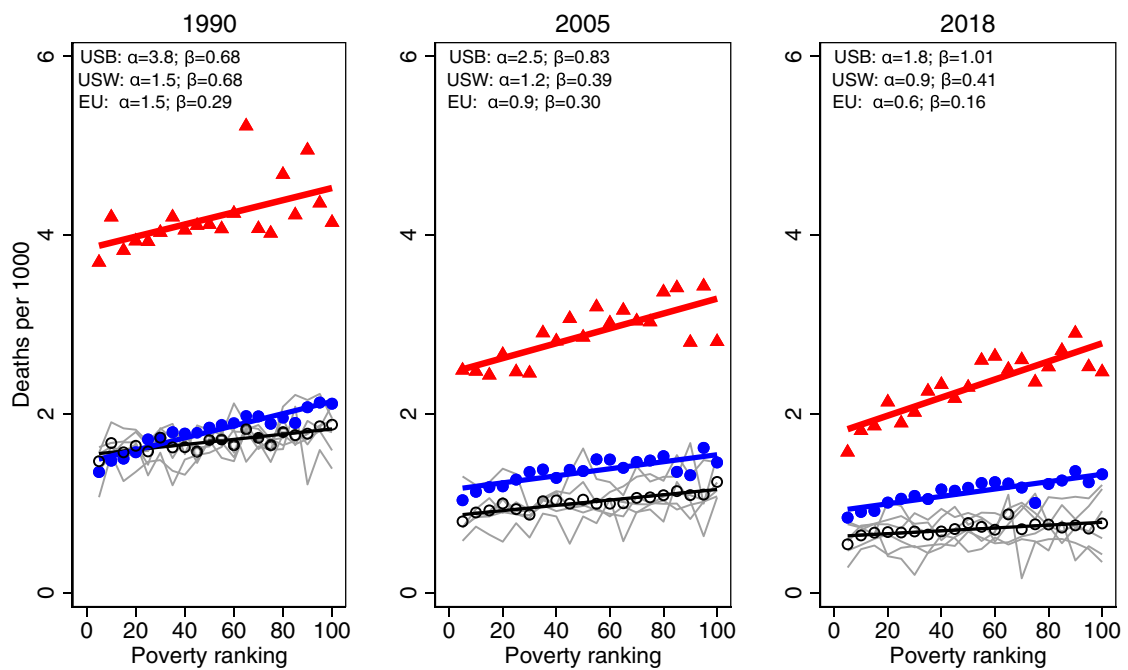

\section{$\Delta$ U.S. Black • U.S. White $\circ$ Europe (ENG/FR/GER/NL/NO/SP)}

Fig. 1. One-year mortality for Black Americans, White Americans, and six European countries, ages 0 to 4, for (Left) 1990, (Middle) 2005 , and (Right) 2018. Average 1-y mortality rates are plotted across poverty rate percentiles. For US Black (USB) and US White (USW) mortality, each bin represents a group of counties with about $5 \%$ of the overall population in the respective year. Black circles show population-weighted average mortality rates across England (ENG), France (FR), Germany (GER), the Netherlands (NL), Norway (NO), and Spain (SP), and each circle represents a group of municipalities or districts representing $5 \%$ of the overall population of each country in the respective year. Gray lines show mortality for each European country (see SI Appendix, Figs. S5-S9 for colorized figures with an extended set of European countries). Germany and Spain are included with 2016 data in Right. Straight lines provide linear fits. $\alpha$ and $\beta$ refer to the fitted lines' intercepts and slopes, respectively. Additional numerical values are reported in SI Appendix, Table S1.

show the trajectory that might have been possible in a high-income country like the United States.

Fig. 4 shows trends for older adults (aged 65 to 79). This figure shows that mortality declined for both Black and White older adults in high- and low-income areas of the United States. Nevertheless, in the lowest-income areas, White American older adults went from having essentially the same mortality rates as Europeans in 1990 to having significantly higher rates in 2018: 27 per 1,000 in the United States compared to 20 per 1,000 in Europe. The mortality rate for Black American older adults aged 65 to 79 in low-income areas was even higher in 2018, at 32 per 1,000. We do not show mortality trends for adults older than age 80. For this group, we are lacking

Age 5-19
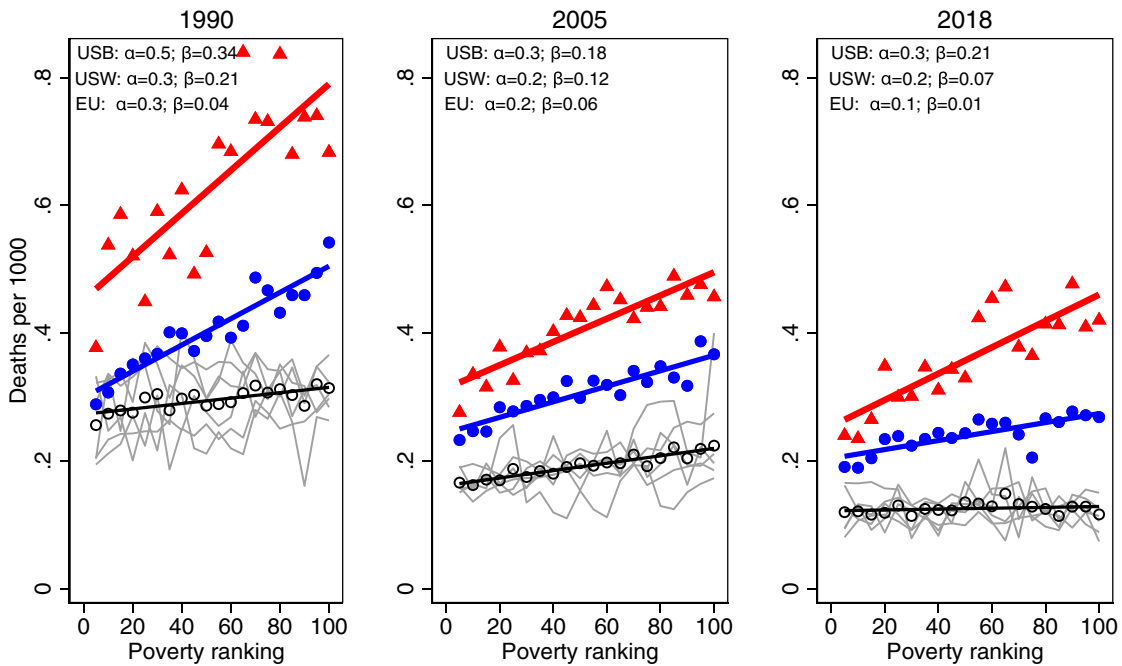

$\Delta$ U.S. Black $\bullet$ U.S. White $\circ$ Europe $(E N G / F R / G E R / N L / N O / S P)$

Fig. 2. One-year mortality for Black Americans, White Americans, and six European countries, ages 5 to 19, for (Left) 1990 , (Middle) 2005, and (Right) 2018. Straight lines provide linear fits. $\alpha$ and $\beta$ refer to the fitted lines' intercepts and slopes, respectively. For further notes, see Fig. 1 . Numerical values and the slopes of fitted lines are reported in SI Appendix, Table S2. 

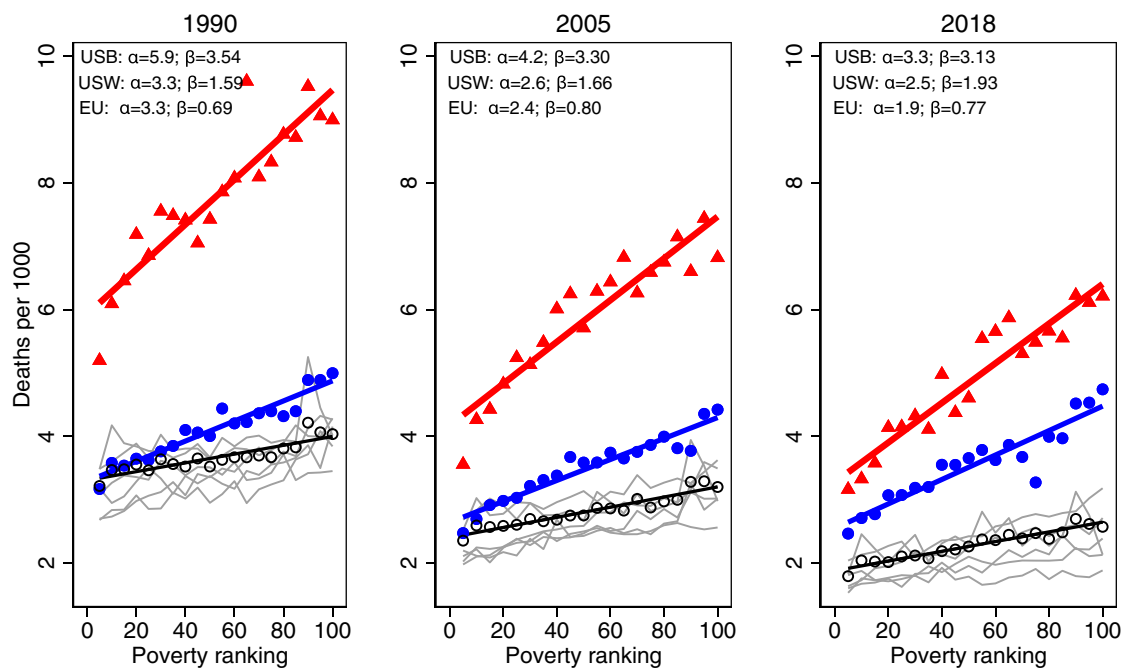

$$
\Delta \text { U.S. Black } \bullet \text { U.S. White } \circ \text { Europe (ENG/FR/GER/NL/NO/SP) }
$$

Fig. 3. One-year mortality for Black Americans, White Americans, and six European countries, ages 20 to 64, for (Left) 1990 , (Middle) 2005, and (Right) 2018. Straight lines provide linear fits. $\alpha$ and $\beta$ refer to the fitted lines' intercepts and slopes, respectively. For further notes, see Fig. 1 . Numerical values and the slopes of fitted lines are reported in SI Appendix, Table S3.

the detailed data on death rates by single year of age to age-adjust the death rates, which is crucial to compare mortality across countries and over time.

Fig. 5 summarizes the trends in age-specific mortality by showing life expectancy at birth for Black and White persons for each year from 1990 to 2018. We have also drawn a trend line using data from 1990 through 2012. This figure highlights the strong convergence between Black and White American mortality rates between 1990 and 2012. Over this period, White American life expectancy continued to improve, but Black American life expectancy improved faster.

Fig. 5 shows that if mortality had continued to evolve at the same rate after 2012 as it did from 1990 to 2012, the gap in life expectancy between Black and White persons would have closed by 2036 . However, improvements in life expectancy among both Black and White Americans faltered after about 2014. Both US White and Black American life expectancy plateaued and then fell between 2015 and 2018. Moreover, the decline in life expectancy

\section{Age 65-79}
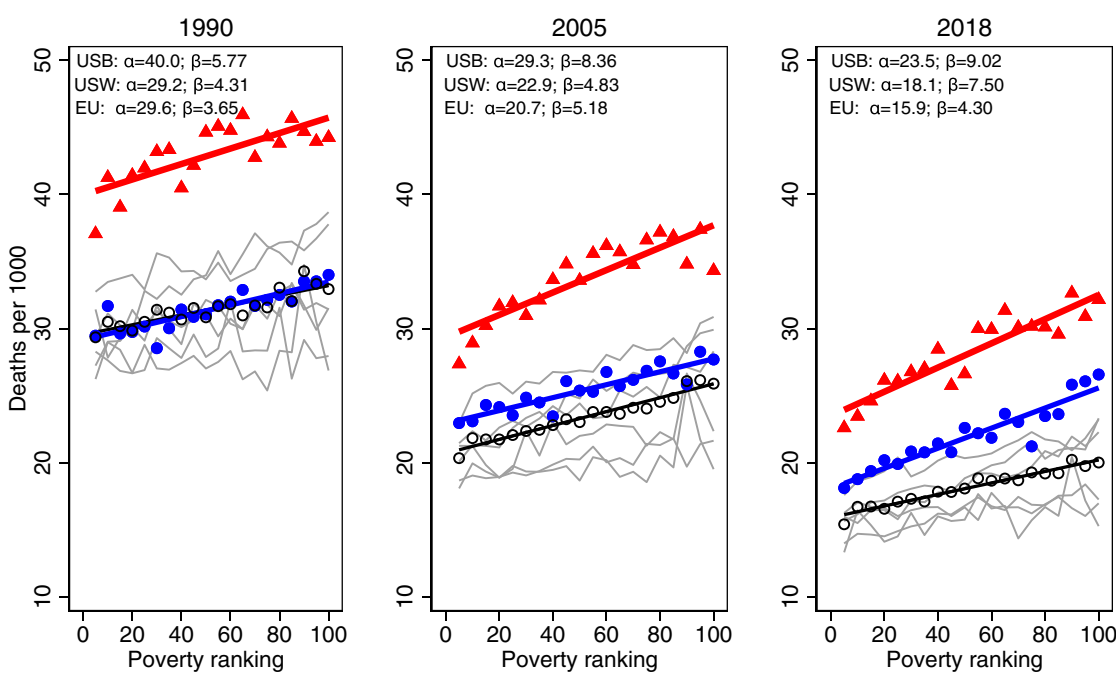

$$
\Delta \text { U.S. Black } \bullet \text { U.S. White } \circ \text { Europe (ENG/FR/GER/NL/NO/SP) }
$$

Fig. 4. One-year mortality for Black Americans, White Americans, and six European countries, ages 65 to 79 , for (Left) 1990 , (Middle) 2005, and (Right) 2018 Straight lines provide linear fits. $\alpha$ and $\beta$ refer to the fitted lines' intercepts and slopes, respectively. For further notes, see Fig. 1 . Numerical values and the slopes of fitted lines are reported in SI Appendix, Table S4. 


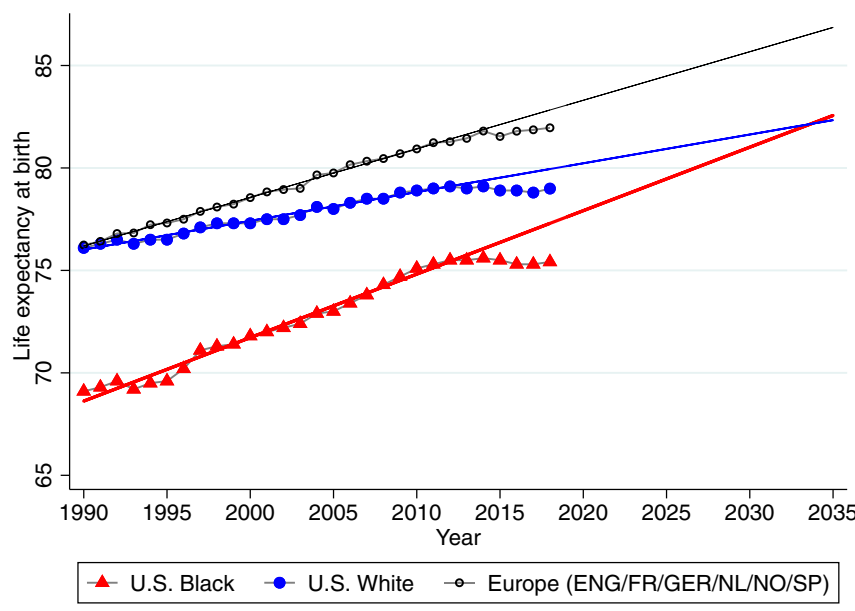

Fig. 5. Life expectancy for Black Americans, White Americans, and six European countries, extrapolated to 2035 fitting a linear trend through 1990 to 2012. Black American, White American, and European life expectancies are plotted over time and extrapolated to 2035 using a linear trend through 1990 to 2012. Black circles show the population weighted average life expectancy across England, France, Germany, the Netherlands, Norway, and Spain.

among Black Americans since 2015 appears to be even more severe than the decline among White Americans. Hence, although some observers have focused on the implications of the COVID-19 pandemic for Black/White American differences (40), even prepandemic, progress in improving the longevity of Black Americans and eliminating racial gaps in life expectancy had started to reverse.

A comparison with European mortality offers a useful perspective. In 1990, life expectancy among White Americans was the same as in the European benchmark countries. However, over the next 3 decades, White Americans increasingly fell behind Europeans. At the same time, life expectancy for Black Americans started far below both European and White American rates in 1990 but grew at a faster rate than European life expectancy.
Although European life expectancy remained above US life expectancy in 2018, European life expectancy also declined relative to the 1990 to 2012 trend after 2014, suggesting that there may be some element common to the United States and Europe that has moderated the growth of life expectancies in most highincome countries. It has been shown that the flattening of life expectancy in the United States was driven primarily by the plateauing of mortality improvements due to cardiovascular disease (41), and this may also be true in Europe.

Fig. 6 offers a closer look at the evolution of racial gaps in mortality by geographic area. As before, counties are sorted into population ventiles by overall poverty rate, but we focus on period life expectancy to summarize mortality rates across all ages. Each ventile represents approximately the same overall population, but race-specific populations are not balanced-in particular, Black people are overrepresented in the lower-income areas and underrepresented in the higher-income areas. This figure traces out the implications of that imbalance for the evolution of life expectancy. Fig. $6 A$ shows the change in race-specific life expectancy between 1990 and 2018, calculated within each ventile. This panel confirms the evidence from Figs. 1-4 that between 1990 and 2018, Black American mortality declined faster than White mortality in all areas. The gap is larger in some ventiles than others but is sizeable in all but the highest-income $5 \%$ of counties. Fig. $6 \mathrm{~B}$ confirms that Black people are overrepresented in lower-income counties and underrepresented in higher-income ones. Fig. $6 C$ shows the contribution of each ventile group to overall life expectancy between 1990 and 2018 for Black and White Americans separately. In other words, Fig. $6 C$ illustrates the impact on life expectancy if only mortality in a given ventile were allowed to change. Fig. $6 C$ shows that improvements in the lowest-income counties made a disproportionate contribution to Black persons life expectancy gains.

Fig. $6 D$ shows the contribution of each ventile to the reduction of the racial mortality gap. Life expectancy in the highest-income counties increased the racial life expectancy gap, not because Black Americans living in these places experienced smaller life expectancy gains than White Americans but because White Americans were overrepresented in high-income areas. In other words, mortality
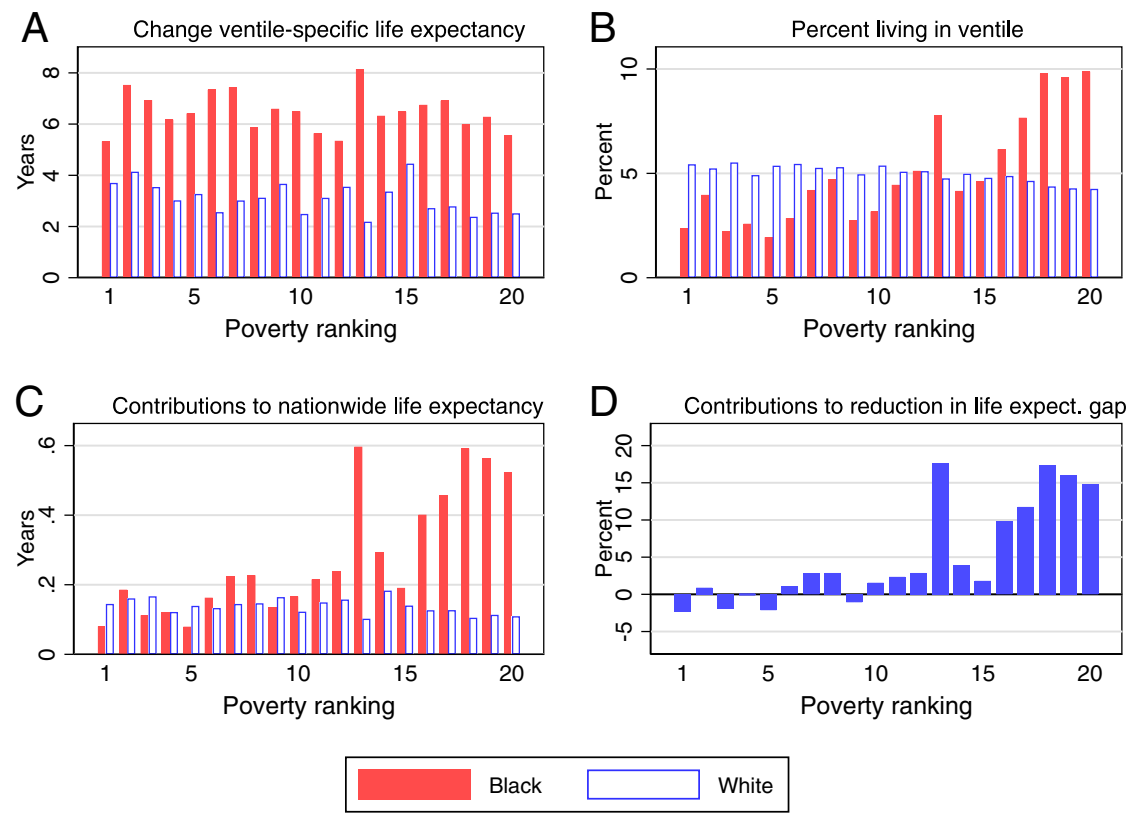

Fig. 6. Population distribution in 1990 and life expectancy contribution for 1990 to 2018 , by ventile and race. (A) The change in race-specific life expectancy calculated within each ventile between 1990 and 2018. (B) The percent of the overall US Black and White population living in each ventile in 1990. (C) The contribution of the mortality changes in each ventile to the countrywide race-specific life expectancy. $(D)$ The percent contribution of the mortality changes in each ventile to the reduction in the life expectancy gap between Black and White Americans. 
improvements among Black Americans living in the highestincome counties had relatively little impact on overall life expectancy of Black people because fewer Black people enjoyed them, while the opposite was the case for White Americans. The result is an increase in the racial life expectancy gap. Similarly, the lowestincome areas contributed the most to life expectancy gains among Black people and to the closing of the racial life expectancy gap because Black Americans are overrepresented in these areas. These results suggest that given continuing overrepresentation of Black Americans in low-income places, improving life expectancy in these places is key to further reductions in racial life expectancy gaps.

SI Appendix, Fig. S1 offers a breakdown of the causes of death that were most responsible for the reduction in the Black-White American mortality gap. These results are subject to the usual caveats about the limitations of cause of death data, particularly when making comparisons over time (42). SI Appendix, Fig. S1A breaks out some of the most important contributors to changes in life expectancy separately for Black and White Americans. One can immediately see that cardiovascular disease and cancer are the most important individual contributors for both groups.

SI Appendix, Fig. S1B shows the percent contribution of each cause to the reduction of the racial difference in life expectancy. SI Appendix, Table S5 shows the corresponding numerical values. SI Appendix, Fig. S1B shows that cardiovascular mortality had the smallest impact on the gap across all causes because reductions in deaths from cardiovascular disease benefitted White and Black persons fairly similarly over this time period. The causes that contributed most to reductions in the gap were, in order of importance, so called "deaths of despair" (43) (16.18\%; this category includes suicide, overdoses, and cirrhosis), cancer (15.96\%), homicide (12.51\%), deaths from causes originating in the fetal or infant period $(11.05 \%)$, and HIV (9.89\%). The importance of overdose deaths (44), homicide, and HIV in explaining racial differences in life expectancy has been previously noted $(3-5,45-48)$. However, it is notable in light of previous work that over the period we analyze, changes in mortality due to cardiovascular disease explain relatively little of the changing gap. This finding indicates that the role of cardiovascular mortality in closing the inequality gap has declined in the 2010s. Faster reductions in cancer deaths among Black Americans also seem to have had a larger impact than they did before 2010 (3-5).

Since the opioid epidemic is one of the most important causes of recent declines in US life expectancy relative to other countries (44), SI Appendix, Fig. S2A compares actual life expectancy with a counterfactual life expectancy computed by assuming that the rate of deaths due to drug overdoses had remained at its 1990 value. SI Appendix, Fig. S2B is similar but assumes that the broader category of deaths of despair, that is, deaths from drug overdose, suicides, and chronic liver disease, had not changed since 1990. SI Appendix, Fig. S2 shows that without drug overdoses, there would still have been a slight downturn in life expectancy around 2015 and 2016 but that life expectancy may well have continued upward after that, albeit with the flattening trend noted above. Comparing $S I$ Appendix, Fig. S2 $A$ and $B$ shows that suicides and chronic liver disease are also important for White Americans, but for Black Americans, only overdoses have had a large impact in terms of life expectancy, and then only since about 2014.

SI Appendix, Fig. S3 and Table S7 provide a similar breakdown of which age groups contributed the most to the decline in the life expectancy gap between Black and White Americans. Consistent with the analysis by cause, the age groups that contributed the most are 0- to 4-y-olds and 20- to 64-y-olds, although this pattern varies somewhat over time (SI Appendix, Table S6). For example, 0 - to 4 -y-olds contributed $19.6 \%$ of the reduction between 1990 and 2000 and $9.83 \%$ between 2000 and 2018. The decomposition shows that the single years of age that contributed most to the closing of the gap were in infancy and among primeage adults (aged 20 to 64). For Black Americans, contributions to improvements in life expectancy rose from age 20 to about age
65 and then declined. As has been noted in the literature (9-11, 49 ), this is strikingly not the case for White Americans, who experienced small declines in life expectancy from about ages 25 to 40 , followed by only small gains to age 60 .

Following Macinko and Elo (50), we also provide a breakdown of differences between Black and White Americans in preventable causes of death below age 65 in SI Appendix, Fig. S4 and Table S8 (all deaths are classified as in ref. 50, reported in SI Appendix, Table S9). SI Appendix, Fig. S4 indicates that Black Americans made major gains to life expectancy in terms of reductions in deaths from causes amenable to medical care and, to a lesser extent, from deaths amenable to intervention. These results indicate a continuation of the trends reported up to 2005 by Macinko and Elo (50), with the exception of ischemic heart disease, which decreased in relevance.

\section{Discussion}

We focus on the evolution of mortality in the 3 decades leading up to the COVID-19 pandemic in order to take stock of the improvements and remaining inequalities that were present in the United States before the pandemic struck. We view racial disparities through the lens of place, comparing gaps in the highestincome parts of the country to those in the lowest-income areas, and we use European mortality rates as a benchmark for assessing mortality differences and trends in those differences.

In 1990, there were remarkable mortality differences between Black and White Americans. For most age groups, Black Americans living in the highest-income US areas had substantially higher mortality rates than White Americans in the country's lowestincome areas. The mortality disadvantage of Black Americans in 1990 was even more pronounced when compared to European countries, while mortality rates of White Americans were fairly similar to those in Europe.

Since 1990, Black Americans experienced large mortality improvements across all age ranges and in both higher- and lowerincome areas, although because Black Americans are more likely to live in lower-income counties, gains in these counties played an outsized role in reducing the racial life expectancy gap. These reductions in mortality were strong enough to reduce the racial mortality gap by $48.9 \%$, despite the fact that White Americans also experienced mortality improvements. Between 1990 and 2018, the US Black-White American life expectancy gap decreased from 7.0 to $3.6 \mathrm{y}$, while the gap between the six European countries and Black Americans decreased from 7.1 to $6.5 \mathrm{y}$.

Mortality improvements among Black Americans and the closing of the racial mortality gap stalled after 2012. Moreover, despite mortality improvements since 1990, White Americans have increasingly lost ground compared to Europeans, with substantial gaps in mortality rates opening between Europeans and White Americans. The gap between Black Americans and the six European countries included decreased by $19 \%$ between 1990 and 2012 but only by $8.3 \%$ in the overall period from 1990 and 2018. Hence, the convergence in the US Black-White American mortality gap reflects real progress among Black Americans even relative to a non-US benchmark, but this progress has reversed after 2012 .

The diverging mortality experience between the United States and Europe is especially evident when analyzing a larger set of nine European countries, although some of these countries are missing data for 1990 (SI Appendix, Figs. S5-S9). Despite strong differences in social and economic starting points across these European countries, by 2018 their mortality gradients fall into a narrow band. Even countries like Portugal, which was much lower income than the European average in 1990, or the Czech Republic, which experienced the fall of the Soviet Union, were able to catch up to higher-income and more stable European countries in terms of mortality rates by 2018. European mortality rates in 2018 lie below US White American mortality rates for each country and across high- and low-income areas. This US health disadvantage 
even among economically advantaged groups in high-income US locations has also been shown for a broad set of health conditions $(51,52)$.

Another remarkable observation is how flat mortality gradients are at younger ages across all European countries. This pattern shows that health improvements among infants, children, and youth have been disseminated within European countries in a way that includes even the lowest-income areas. It suggests that there is great potential for disadvantaged infants, children, and youth living in lower-income US areas to catch up to European standards.

Focusing on the disparity between Black and White Americans, we show that improvements in Black Americans' relative to White Americans' life expectancy in the lowest-income counties had the greatest impact in narrowing the gap overall. In terms of mortality causes driving these improvements, greater reductions in Black relative to White American death rates due to cancer, homicide, $\mathrm{HIV}$, and causes originating in the fetal or infant period had the largest impacts, while smaller increases in Black American compared to White American deaths of despair also closed the gap. Consistent with the importance of these causes, we find that rapid reductions in Black relative to White American deaths in early childhood and prime-aged adults (aged 20 to 64) accounted for the majority of the closure of the gap. Consistent once again with the importance of these causes (49), deaths due to causes amenable to medical care showed greater reductions for Black Americans relative to White Americans, greatly contributing to the closure of the gap in life expectancy. Reductions in causes amenable to intervention also played an important role, in line with prior research studying longitudinal racial disparities in more nuanced health indicators (53).

Many authors have commented on the role of systemic racism in shortening Black relative to White American life expectancies in the United States (54-56). Unpacking some of the dimensions of racism suggests that there are many possible reasons for these broad improvements in the health of Black Americans. The literature on the relationship between education and health suggests that improvements in the quantity and quality of education available to Black children and young adults over the past decades is one possible contributor to improved longevity and reduced gaps in life expectancy spread broadly over prime-aged adults (aged 20 to 64$)(57,58)$. Our results suggest that improvements in the availability of medical care are also likely to have been important in reducing racial disparities in mortality (59). Health care developments that may have been particularly important include expansions of the Medicaid program to cover pregnant women and children starting in the 1990 s, which likely account for much of the improvement among infants (60), as well as improved access to treatment for cancer and HIV. Long-term health effects of access to Medicaid as well as other safety net programs such as food stamps and the Earned Income Tax Credit may also be an important contributor to mortality reductions (61). Reductions in pollution may have played a role given that Black Americans are more likely than White Americans to live in more-polluted areas (62-64).

Despite the strong mortality improvements among Black Americans over the past 3 decades, a dramatic gap remains, and this gap has increased again in recent years. It is important to understand which medical, social, and policy developments helped to increase the longevity of Black Americans through 2012 and how these positive changes can be reinforced over the coming decades with the ultimate goal of fully closing the racial longevity gap in the United States. Moreover, the comparisons with Europe suggest that mortality rates of both Black and White Americans could fall much further across all ages and across higher- and lower-income areas.

\section{Materials and Methods}

US Mortality. US Black and White American mortality rates are constructed by dividing death counts by population estimates for single years of age, county, and calendar year. Death counts come from the US Vital Statistics mortality files, while population estimates are provided by the National Center for Health Statistics (NCHS). The NCHS estimates are "bridged"; that is, they convert multiple race categories reported in the 2000 and 2010 Censuses back to single race categories comparable with those reported on the death certificates. Throughout the paper, "Black" populations include both non-Hispanic and Hispanic Black persons, while "White" populations include both non-Hispanic and Hispanic White persons. Neither race group includes American Indian and Alaska Native, Asian, or Native Hawaiian and Other Pacific Islander (the NCHS notes that these race categories "represent a social-political construct and are not anthropologically or biologically based") (65). Mortality rates spanning multiple years of age are age-adjusted using the 2015 US population. We ageadjust because in an age bracket such as ages 65 to 79, a group with more 79-y-olds would be expected to have higher mortality.

US Poverty Ranking. As in ref. 25, we rank counties by their poverty rate and place them into groups of fixed population size. This allows us to analyze trends in age-specific mortality across areas ranked by an area's poverty rate while taking into account population shifts across areas. We rank all US counties in 1990, 2005, and 2018 by their poverty level and then divide them into 20 groups, each representing roughly $5 \%$ of the overall US population. This way we can compare, for example, the $5 \%$ of the population living in the lowest-income counties in 1990 with the $5 \%$ of the population living in the highest-income counties in 1990 and analyze how the mortality differences between these groups change over time. We refer to the county groups with the highest (lowest) fractions of their populations in poverty as the lowest-income (highest-income) counties. Our approach reassigns county groups in 1990, 2005, and 2018 to adjust for changes in county ranking and population size. Poverty rates are taken from the 1990 and 2000 Censuses and the 2014 to 2018 American Community Survey 5-y sample and interpolated for intermediate years.

European Mortality. Data for nine European countries (Czech Republic, England, Finland, France, Germany, the Netherlands, Norway, Portugal, and Spain) come from the Institute for Fiscal Studies (IFS) project on Geographic Approaches to Inequalities in Mortality (66) and are treated similarly. Additional details on data sources are provided in SI Appendix.

Figs. 1-4 include the six European countries that provide consistent mortality data from 1990 onward (England, France, Germany, the Netherlands, Norway, and Spain). Figs. 1-4 include a mean Europe mortality rate, representing the population-weighted average of mortality rates across these countries in each ventile. SI Appendix, Figs. S5-S9 show analogous figures using all nine of the European countries in the IFS study. See $S I$ Appendix for further information about these countries.

Mortality rates across all countries and years are age-adjusted using the 2015 US population, based on 5-y age groups. The following describes the respective area definitions, ranking measures, and available data years for each of the European countries included in Figs. 1-4: England, local authorities ranked by a deprivation index, for 1992 to 2017; France, départements ranked by poverty rate, for 1990 to 2018; Germany, districts ranked by per-capita income in 1990, 2005, and 2016 (1990 excludes East Germany because of the exceptionally high mortality in East Germany around the time of reunification); the Netherlands, municipalities ranked by poverty rate, 1995, 2005, and 2016; Norway, municipalities ranked by poverty rate, 1990 to 2018; and Spain, municipalities ranked by median income, 1990 to 2016 . Further details on area definitions and the poverty or deprivation variables used for ranking areas can be found in ref. 51 .

Life Expectancy Data. We construct US Black and White American life expectancy at birth based on 1-y mortality rates for the years 1990 to 2018 . The Human Mortality Database (67) provides life expectancy estimates for the European countries in our study, while life expectancy estimates for England are provided by the United Kingdom Office for National Statistics.

Decompositions. We offer breakdowns of the contributions of location, age, and cause of death to Black and White American life expectancy and to the closing of the racial mortality gap. Each breakdown is based on asking how life expectancy would have changed if all other factors besides the one being considered had remained constant at their 1990 levels. For example, we ask how life expectancy would have changed between 1990 and 2018 if only the 
homicide rate had fallen while all other causes remained at their 1990 values. In all cases, this hypothetical exercise is conducted separately for Black and White Americans. Letting only one mortality rate change while keeping all other rates constant understates the one rate's overall contribution to life expectancy if other rates also improved. The reason is that the life expectancy formula interacts mortality rates at all ages. For example, a higher survival rate in old age makes improvements in infant mortality more valuable and vice versa. Our hypothetical life expectancy measure ignores interaction effects because they cannot be assigned to a specific age. Hence, our results should be interpreted as the partial effect of a given factor, which is sometimes referred to as the exclusive life expectancy impact of an age-specific effect (68). For further details, see SI Appendix.

1. C. J. L. Murray et al., Eight Americas: Investigating mortality disparities across races, counties, and race-counties in the United States. PLoS Med. 3, e260 (2006).

2. M. S. Shiels et al., Trends in premature mortality in the USA by sex, race, and ethnicity from 1999 to 2014: An analysis of death certificate data. Lancet 389, 1043-1054 (2017).

3. S. Harper, J. Lynch, S. Burris, G. Davey Smith, Trends in the black-white life expectancy gap in the United States, 1983-2003. JAMA 297, 1224-1232 (2007).

4. N. E. Adler, D. H. Rehkopf, U.S. disparities in health: Descriptions, causes, and mech anisms. Annu. Rev. Public Health 29, 235-252 (2008).

5. S. Harper, D. Rushani, J. S. Kaufman, Trends in the black-white life expectancy gap, 2003-2008. JAMA 307, 2257-2259 (2012).

6. J. Currie, H. Schwandt, Mortality inequality: The good news from a county-level approach. J. Econ. Perspect. 30, 29-52 (2016)

7. A. Case, A. Deaton, Life expectancy in adulthood is falling for those without a BA degree, but as educational gaps have widened, racial gaps have narrowed. Proc. Natl. Acad. Sci. U.S.A. 118, e2024777118 (2021)

8. B. Bosworth, K. Zhang, Evidence of increasing differential mortality: A comparison of the HRS and SIPP. SSRN [Preprint] (2015). https://doi.org/10.2139/ssrn.2625792 (Accessed 14 September 2021).

9. A. Case, A. Deaton, Rising morbidity and mortality in midlife among white non Hispanic Americans in the 21st century. Proc. Natl. Acad. Sci. U.S.A. 112, 15078-15083 (2015).

10. A. Case, A. Deaton, Mortality and morbidity in the $21^{\text {st }}$ century. Brookings Pap. Econ Act. 2017, 397-476 (2017)

11. A. Case, A. Deaton, Deaths of Despair and the Future of Capitalism (Princeton University Press, 2020).

12. R. Chetty et al., The association between income and life expectancy in the United States, 2001-2014. JAMA 315, 1750-1766 (2016).

13. D. M. Cutler, F. Lange, E. Meara, S. Richards-Shubik, C. J. Ruhm, Rising educationa gradients in mortality: The role of behavioral risk factors. J. Health Econ. 30 1174-1187 (2011)

14. E. R. Meara, S. Richards, D. M. Cutler, The gap gets bigger: Changes in mortality and life expectancy, by education, 1981-2000. Health Aff. (Millwood) 27, 350-360 (2008).

15. J. K. Montez, L. F. Berkman, Trends in the educational gradient of mortality among US adults aged 45 to 84 years: Bringing regional context into the explanation. Am. J. Public Health 104, e82-e90 (2014).

16. J. K. Montez, A. Zajacova, Explaining the widening education gap in mortality among U.S. white women. J. Health Soc. Behav. 54, 166-182 (2013).

17. National Academies of Sciences, Engineering, and Medicine et al., The Growing Gap in Life Expectancy by Income: Implications for Federal Programs and Policy Responses (National Academies Press, 2015)

18. S. J. Olshansky et al., Differences in life expectancy due to race and educational differences are widening, and many may not catch up. Health Aff. (Millwood) 31, 1803-1813 (2012)

19. G. Pappas, S. Queen, W. Hadden, G. Fisher, The increasing disparity in mortality between socioeconomic groups in the United States, 1960 and 1986. N. Engl. J. Med. 329, 103-109 (1993).

20. J. Pijoan-Mas, J.-V. Ríos-Rull, Heterogeneity in expected longevities. Demography $\mathbf{5 1}$ 2075-2102 (2014)

21. S. H. Preston, I. T. Elo, Are educational differentials in adult mortality increasing in the United States? J. Aging Health 7, 476-496 (1995)

22. H. Waldron, Mortality differentials by lifetime earnings decile: Implications for evaluations of proposed Social Security law changes. Soc. Secur. Bull. 73, 1-37 (2013)

23. A. S. Venkataramani, R. O'Brien, A. C. Tsai, Declining life expectancy in the United States: The need for social policy as health policy. JAMA 325, 621-622 (2021).

24. M. Baker, J. Currie, H. Schwandt, Mortality inequality in Canada and the United States: Divergent or convergent trends? J. Labor Econ. 37, S325-S353 (2019).

25. J. Currie, H. Schwandt, Inequality in mortality decreased among the young while increasing for older adults, 1990-2010. Science 352, 708-712 (2016).

26. J. Currie, H. Schwandt, J. Thuilliez, Pauvreté, Egalité, Mortalité: Mortality (in)equality in France and the United States. J. Popul. Econ. 33, 197-231 (2020).

27. M. Ezzati, A. B. Friedman, S. C. Kulkarni, C. J. L. Murray, The reversal of fortunes: Trends in county mortality and cross-county mortality disparities in the United States. PLoS Med. 5, e66 (2008)

28. N. Krieger et al., The fall and rise of US inequities in premature mortality: 1960-2002. Plos Med. 5, e46 (2008).
Data Availability. All datasets used in this study are publicly available online or can be obtained from the National Vital Statistics offices. See SI Appendix, section $\mathrm{C}$, for additional details and download links.

ACKNOWLEDGMENTS. We thank J.B., S.K., and K.G.S. for organizing the IFS working group on geographical approaches to measuring inequality in mortality, with financial support from the Economic and Social Research Council Centre for the Microeconomic Analysis of Public Policy at IFS (Grant ES/T014334/1). C.C received support from the Science and Technology Foundation (Fundação para a Ciência e a Tecnologia [FCT]), the European Social Fund, and the Centro Operational Programme (Grant SFRH/BD/132218/2017). P.S. received support from the Centre of Studies in Geography and Spatial Planning (Grant UIDB/04084/2020), through an FCT fund.

29. H. Wang, A. E. Schumacher, C. E. Levitz, A. H. Mokdad, C. J. Murray, Left behind: Widening disparities for males and females in US county life expectancy, 1985-2010. Popul. Health Metr. 11, 8 (2013)

30. G. K. Singh, M. Siahpush, Widening socioeconomic inequalities in US life expectancy, 1980-2000. Int. J. Epidemiol. 35, 969-979 (2006).

31. Y. C. Vierboom, S. H. Preston, A. S. Hendi, Rising geographic inequality in mortality in the United States. SSM Popul. Health 9, 100478 (2019).

32. J. B. Dowd, A. Hamoudi, Is life expectancy really falling for groups of low socioeconomic status? Lagged selection bias and artefactual trends in mortality. Int J. Epidemiol. 43, 983-988 (2014).

33. A. S. Hendi, Trends in U.S. life expectancy gradients: The role of changing educational composition. Int. J. Epidemiol. 44, 946-955 (2015).

34. T. Goldring, F. Lange, S. Richards-Shubik, Testing for changes in the SES-mortality gradient when the distribution of education changes too. J. Health Econ. 46, $120-130(2016)$

35. A. S. Hendi, I. T. Elo, P. Martikainen, The implications of changing education distributions for life expectancy gradients. Soc. Sci. Med. 272, 113712 (2021).

36. J. R. Wilmoth, C. Boe, M. Barbieri, "Geographic differences in life expectancy at age 50 in the United States compared with other high-income countries" in International Differences in Mortality at Older Ages: Dimensions and Sources, E. M. Crimmins, S. H. Preston, B. Cohen, Eds. (The National Academies Press, 2010), pp. 333-366.

37. J. P. Mackenbach et al., Socioeconomic inequalities in health in 22 European countries. N. Engl. J. Med. 358, 2468-2481 (2008).

38. J. P. Mackenbach et al., Changes in mortality inequalities over two decades: Register based study of European countries. BMJ 353, i1732 (2016).

39. J. Y. Ho, A. S. Hendi, Recent trends in life expectancy across high income countries: Retrospective observational study. BMJ 362, k2562 (2018).

40. T. Andrasfay, N. Goldman, Reductions in 2020 US life expectancy due to COVID-19 and the disproportionate impact on the Black and Latino populations. Proc. Natl. Acad. Sci. U.S.A. 118, e2014746118 (2021).

41. N. K. Mehta, L. R. Abrams, M. Myrskylä, US life expectancy stalls due to cardiovascular disease, not drug deaths. Proc. Natl. Acad. Sci. U.S.A. 117, 6998-7000 (2020).

42. I. T. Elo, G. L. Drevenstedt, Cause-specific contributions to black-white differences in male mortality from 1960 to 1995. Demogr. Res. 2 (Special), 255-276 (2004).

43. A. Case, A. Deaton, Mortality and morbidity in the 21 st century. Brookings Pap. Econ Act. 2017, 397-476 (2017)

44. S. Harper, C. A. Riddell, N. B. King, Declining life expectancy in the United States: Missing the trees for the forest. Annu. Rev. Public Health 42, 381-403 (2021)

45. K. D. Kochanek, J. D. Maurer, H. M. Rosenberg, Why did black life expectancy decline from 1984 through 1989 in the United States? Am. J. Public Health 84 938-944 (1994)

46. M. Roberts, E. N. Reither, S. Lim, Contributors to the black-white life expectancy gap in Washington D.C. Sci. Rep. 10, 13416 (2020).

47. P. Sharkey, M. Friedson, The impact of the homicide decline on life expectancy of African American males. Demography 56, 645-663 (2019).

48. A. Leive, C. Ruhm, Education Gradients in Mortality Trends by Gender and Race (National Bureau of Economic Research, 2021).

49. I. T. Elo, A. S. Hendi, J. Y. Ho, Y. C. Vierboom, S. H. Preston, Trends in Non-Hispanic White mortality in the United States by metropolitan-nonmetropolitan status and region, 1990-2016. Popul. Dev. Rev. 45, 549-583 (2019)

50. J. Macinko, I. T. Elo, Black-white differences in avoidable mortality in the USA, 1980 2005. J. Epidemiol. Community Health 63, 715-721 (2009).

51. E. J. Emanuel et al., Comparing health outcomes of privileged US Citizens with those of average residents of other developed countries. JAMA Intern. Med. 181, 339-344 (2021).

52. J. Banks, M. Marmot, Z. Oldfield, J. P. Smith, Disease and disadvantage in the United States and in England. JAMA 295, 2037-2045 (2006)

53. K. M. Harris, P. Gordon-Larsen, K. Chantala, J. R. Udry, Longitudinal trends in race/ ethnic disparities in leading health indicators from adolescence to young adulthood. Arch. Pediatr. Adolesc. Med. 160, 74-81 (2006).

54. D. R. Williams, C. Collins, US socioeconomic and racial differences in health: Patterns and explanations. Annu. Rev. Sociol. 21, 349-386 (1995).

55. D. R. Williams, P. B. Jackson, Social sources of racial disparities in health. Health Aff (Millwood) 24, 325-334 (2005)

56. J. C. Phelan, B. G. Link, Is racism a fundamental cause of inequalities in health? Annu. Rev. Sociol. 41, 311-330 (2015).

57. I. T. Elo, S. H. Preston, Educational differentials in mortality: United States, 1979-85. Soc. Sci. Med. 42, 47-57 (1996). 
58. D. Frisvold, E. Golberstein, The effect of school quality on black-white health differences: Evidence from segregated southern schools. Demography 50, 1989-2012 (2013).

59. A. Nelson, Unequal treatment: Confronting racial and ethnic disparities in health care. J. Natl. Med. Assoc. 94, 666-668 (2002).

60. A. D. Racine, R. Kaestner, T. J. Joyce, G. J. Colman, Differential impact of recent Medicaid expansions by race and ethnicity. Pediatrics 108, 1135-1142 (2001)

61. H. Hoynes, D. W. Schanzenbach, D. Almond, Long-run impacts of childhood access to the safety net. Am. Econ. Rev. 106, 903-934 (2016).

62. J. Currie, J. Voorheis, R. Walker, What Caused Racial Disparities in Particulate Exposure to Fall?: New Evidence from the Clean Air Act and Satellite-based Measures of Air Quality (National Bureau of Economic Research, 2020).

63. S. Banzhaf, L. Ma, C. Timmins, Environmental justice: The economics of race, place, and pollution. J. Econ. Perspect. 33, 185-208 (2019).
64. J. Currie, Inequality at birth: Some causes and consequences. Am. Econ. Rev. 101, 1-22 (2011).

65. Centers for Disease Control and Prevention (CDC), Documentation for Bridged-Race Vintage 2006 (July 1, 2000 - July 1, 2006) Postcensal Population Estimates for Calculating Vital Rates. https://wonder.cdc.gov/wonder/help/populations/bridged-race/ Estimates2000-06.html. Accessed 14 September 2021.

66. J. Banks, J. Currie, S. Krutikova, K. G. Salvanes, H. Schwandt, The evolution of mortality inequality in 11 OECD countries: Introduction. Fisc. Stud. 42, 9-23 (2021).

67. M. Barbieri et al., Data resource profile: The human mortality database (HMD). Int. J. Epidemiol. 44, 1549-1556 (2015).

68. E. E. Arriaga, Measuring and explaining the change in life expectancies. Demography 21, 83-96 (1984). 